\title{
Costless delay in negotiations
}

\section{P. Jean-Jacques Herings ${ }^{1}$ (D) $\cdot$ Harold Houba $^{2,3}$}

Received: 16 September 2019 / Accepted: 31 May 2021 / Published online: 9 June 2021

(c) The Author(s) 2021

\begin{abstract}
We study bargaining models in discrete time with a finite number of players, stochastic selection of the proposing player, endogenously determined sets and orders of responders, and a finite set of feasible alternatives. The standard optimality conditions and system of recursive equations may not be sufficient for the existence of a subgame perfect equilibrium in stationary strategies (SSPE) in case of costless delay. We present a characterization of SSPE that is valid for both costly and costless delay. We address the relationship between an SSPE under costless delay and the limit of SSPEs under vanishing costly delay. An SSPE always exists when delay is costly, but not necessarily so under costless delay, even when mixed strategies are allowed for. This is surprising as a quasi SSPE, a solution to the optimality conditions and the system of recursive equations, always exists. The problem is caused by the potential singularity of the system of recursive equations, which is intimately related to the possibility of perpetual disagreement in the bargaining process.
\end{abstract}

Keywords Bargaining - Subgame perfect equilibrium · Stationary strategies · Existence $\cdot$ Costless delay

JEL Classification $\mathrm{C} 72 \cdot \mathrm{C} 73 \cdot \mathrm{C} 78$

The authors thank János Flesch, Dinard van der Laan, Ad Ridder, Klaus Ritzberger and Eilon Solan and several participants of the Conference on Economics Design 2009, the Conference of the Society for the Advancement of Economic Theory 2011, GAMES 2012, SAET 2013, the World Congress of the Econometric Society 2015 and GAMES 2016 for valuable comments.

$凶 \quad$ P. Jean-Jacques Herings

P.Herings@maastrichtuniversity.nl

Harold Houba

harold.houba@vu.nl

1 Department of Economics, Maastricht University, P.O. Box 616, 6200 MD Maastricht, The Netherlands

2 Department of Econometrics, Vrije Universiteit Amsterdam, De Boelelaan 1105, 1081 HV Amsterdam, The Netherlands

3 Tinbergen Institute, Amsterdam, The Netherlands 


\section{Introduction}

Strategic bargaining theory, boosted by the contribution of Rubinstein (1982), has contributed significantly to our understanding of negotiation processes. For surveys of the extensive literature on strategic bargaining, we refer the reader to the surveys by Osborne and Rubinstein (1990), Muthoo (1999), Houba and Bolt (2002), Ray (2007) and Eraslan and Evdokimov (2019).

An important role in bargaining theory is played by the costs of delay, either because the future is discounted or because there is a positive probability that negotiations break down before an agreement is reached. Many influential contributions analyze negotiations under vanishing costs of delay as well as the limit case of costless delay. Costless delay is analyzed in, e.g., Binmore (1986), Baron and Ferejohn (1989), Perry and Reny (1994), Moldovanu and Winter (1995), Bloch (1996), Banks and Duggan (2000), Dàvila and Eeckhout (2008), Horniaček (2008) and Herings and Houba (2016).

In this paper, we present a characterization of subgame perfect equilibria in stationary strategies (SSPE) for bargaining models that is valid for both the cases of costly and costless delay. We also address the relationship between SSPE under costless delay and the limit of SSPEs under vanishing costly delay. Our analysis is inspired by a simple example of hedonic coalition formation with costless delay where an SSPE does not exist, even when mixed strategies are allowed for. In this example, the limit of SSPEs under vanishing costly delay is a strategy profile that fails to be an SSPE of the model with costless delay. Our study has been influenced by Magill and Quinzii (2003). In this reference, a recursive stochastic overlapping-generations model is first analyzed under both certainty and uncertainty before vanishing uncertainty is investigated.

Our bargaining model is as follows. At the beginning of any bargaining round, the proposer specifies a proposal, which consists of an alternative, a set of players that can implement this alternative, and an order in which the players in this set respond. The first rejection by a responding player ends the current bargaining round. Next a chance move determines whether negotiations break down or continue and, conditional on continuation, who will be the next proposer. Our bargaining protocol covers special cases that have been studied in the literature before like a fixed rotating orders of proposers, time-invariant selection probabilities, and the rejecter-becomesproposer protocol. Our model can be used to study unanimity bargaining, coalitional bargaining, and legislative bargaining.

The standard characterization of an SSPE under costly delay consists of optimality conditions corresponding to the one-stage deviation principle together with a system of recursive equations to pin down equilibrium utilities. This characterization provides necessary but not sufficient conditions in case delay is costless. A strategy profile that satisfies the conditions in this characterization is therefore called a quasi SSPE. We show that a quasi SSPE always exists, also when delay is costless. In a motivating example, we compute the quasi equilibria in a game of hedonic coalition formation as proposed in Bloch and Diamantoudi (2011). When delay is costly, the quasi SSPE is an SSPE. The limit of SSPEs when costs of delay vanish leads to the unique quasi SSPE for the model with costless delay. Nevertheless, this quasi SSPE fails to be an SSPE for the model with costless delay. 
We show that under costless delay the system of recursive equations can be singular and may admit solutions that do not correspond to the actual utilities induced by a strategy profile. On the other hand, we show that the utilities generated by a particular strategy profile are always a solution to the system of recursive equations. We present necessary and sufficient conditions such that the system of recursive equations has a unique solution. These conditions are in terms of the finest decomposition of the matrix of state transition probabilities and require that each irreducible block in the finest decomposition has at least one row sum strictly less than one. Next, we apply these conditions to conclude that a quasi SSPE is an SSPE if either delay is costly or delay is costless and perpetual disagreement never occurs. We also present a result that applies when the system of recursive equations is singular. A necessary and sufficient condition such that the solution to the system of recursive equations corresponds to the utilities generated by a strategy profile is that the solution is equal to zero at all states which lead to perpetual disagreement.

After the analysis of the system of recursive equations, we consider the characterization of stationary best responses and of an SSPE. It is shown that there is a stationary best response against any stationary strategy profile. A stationary best response is characterized by the optimality conditions and the system of recursive equations for the responding player together with the requirement that utilities are equal to zero in all states that lead to perpetual disagreement. Combining the characterization of stationary best responses across all players gives a characterization of SSPE.

Our results imply the existence of an SSPE under costly delay. This also follows from standard results on equilibrium existence in stochastic games, see, e.g., Fink (1964), Takahashi (1964) and Sobel (1971). For the case of costless delay, we obtain existence of an SSPE if there is a quasi SSPE which does not lead to perpetual disagreement at any state. If the limit of SSPEs under vanishing costly delay is a strategy profile without perpetual disagreement at any state, then the limit is an SSPE under costless delay. Vice versa, if there is no SSPE under costless delay, then there is a state where delay goes to infinity for any sequence of SSPEs under vanishing costly delay.

The case of costless delay is notoriously difficult, which is caused by the lack of continuity of utility functions. ${ }^{1}$ Non-existence of Nash equilibrium in behavioral strategies has been noted by Blackwell and Ferguson (1968) and has spurred an extensive literature seeking existence of weaker notions of Nash equilibrium and subgame perfect equilibrium in special classes of stochastic games. For $\varepsilon$-Nash equilibria, existence has been shown by Mertens and Neyman (1981) for two-person zero-sum stochastic games and by Vieille (2000a,b,c) for general two-player stochastic games. A general result for stochastic games with three or more players is lacking thus far. Flesch et al. (2010) have demonstrated the existence of a subgame-perfect $\varepsilon$-equilibrium for every $\varepsilon>0$ for the subclass of recursive stochastic games with non-negative utilities, whereas Kuipers et al. (2021) obtain such a result for the subclass of recursive stochastic games with deterministic transitions.

This paper is organized as follows. After the introduction of the bargaining model in Sect. 2, we present the notion of quasi SSPE in Sect. 3. Two motivating examples

\footnotetext{
${ }^{1}$ In perfect information games with continuous utility functions, existence of a subgame-perfect equilibrium follows from results in Harris (1985) and Alós-Ferrer and Ritzberger (2016).
} 
are discussed in Sect. 4. The necessary and sufficient conditions such that the system of recursive equations admits a unique solution and the necessary and sufficient conditions for a solution to the system of recursive equations to correspond to the correct utilities are established in Sect. 5. The necessary and sufficient conditions for a strategy profile to be a stationary best response are derived in Sect. 6. Section 7 presents the characterization of SSPE and investigates the case of vanishing costs of delay. Section 8 contains the conclusion.

\section{The bargaining model}

We study a non-cooperative model of bargaining with a set $N=\{1, \ldots, n\}$ of two or more players who negotiate about the selection of an alternative in a non-empty, finite set of feasible alternatives $A$.

A proposal consists of an alternative and a coalition that can implement the alternative. We assume that the proposer not only selects the coalition, but also the order in which other coalition members respond. Formally, for $C$ a proper subset of $N$, the collection $O(C)$ consists of all permutations of the players in $C$ and the set $O \subset \cup_{\left\{C \in 2^{N}:|C|<n\right\}} O(C)$ consists of all possible responder orders. The set $C$ is allowed to be empty, which makes it possible to model that the proposer can implement an alternative without the consent of any other player. The coalition involved in responder order $o \in O$ is denoted by $C(o)$.

The non-empty set $X^{i} \subset A \times O$ denotes the set of feasible proposals for player $i \in N$. We assume that if $(a, o) \in X^{i}$, then $i \notin C(o)$, since $i$ as a proposer of $(a, o)$ does not have to respond to $(a, o)$. For $j \in C(o)$, we define $k^{j}(o) \in\{2, \ldots,|C(o)|+1\}$ as one plus the rank of $j$ in $o$. It corresponds to the stage of the bargaining round at which player $j$ is active as a responder.

Negotiations proceed in discrete time. At round $t \in \mathbb{N}$, the proposing player $i^{t} \in N$ selects a proposal $x^{t}=\left(a^{t}, o^{t}\right) \in X^{i^{t}}$, after which all players in $C\left(o^{t}\right)$ sequentially and publicly respond in the order described by $o^{t}$. Alternative $a^{t}$ is implemented in round $t$ and bargaining ends if all players in $C\left(o^{t}\right)$ accept.

Otherwise, the first rejection by a player, denoted by $r^{t} \in N$, terminates the current bargaining round. Alternative $a^{t}$ is rejected and round $t$ is concluded with a draw by nature that is modeled as a compound lottery. First, nature decides with probability $\delta \in[0,1]$ whether bargaining proceeds to round $t+1$, where $\delta \in[0,1)$ corresponds to the case with costly delay and $\delta=1$ to costless delay. With complementary probability $1-\delta$ bargaining breaks down and the status quo $q$ results, which, without loss of generality, is assumed not to belong to the set of alternatives $A$. Second, when bargaining proceeds, nature selects player $i \in N$ to be the proposer in round $t+1$ with probability $\rho_{i}\left(i^{t}, x^{t}, r^{t}\right) \in[0,1]$, where $\sum_{i \in N} \rho_{i}\left(i^{t}, x^{t}, r^{t}\right)=1$. Prior to round 1 , nature selects player $i \in N$ to be the proposer in round 1 with probability $\bar{\rho}_{i} \in[0,1]$, where $\sum_{i \in N} \bar{\rho}_{i}=1$.

Several influential bargaining models can be incorporated as special cases of our set-up. In bilateral bargaining $X^{i}=\{(a,(j)) \mid a \in A\}$ expresses that any alternative in $A$ can be proposed by player $i \in N$ and that acceptance by the responding player $j \neq i$ is required for implementation of $a$. More generally, multilateral unanimity 
bargaining can be modeled by taking $X^{i} \subset A \times O(N \backslash\{i\})$. The standard case of an exogenous responder order, say in ascending order, is captured by the requirement that, for every $(a, o) \in X^{i}, o=(1, \ldots, i-1, i+1, \ldots, n)$.

In coalitional bargaining models, the proposer selects a coalition and an alternative that is feasible for the coalition. An alternative is accepted if and only if it is approved by all coalition members. Let $A(C) \subset A$ denote the set of feasible alternatives for coalition $C \subset N$. To capture such coalitional bargaining models, we take $X^{i} \subset\{(a, o) \in A \times O \mid a \in A(\{i\} \cup C(o))\}$. Sometimes, like in Chatterjee et al. (1993), negotiations continue after the formation of a coalition. Our analysis can be extended to this case, but would require the introduction of additional state variables.

We can also incorporate many specifications used in legislative bargaining. The case of a majority rule as in Baron and Ferejohn (1989) is obtained by setting $X^{i} \subset$ $A \times\{o \in O|\{i\} \cup| C(o) \mid>n / 2\}$. In case player $j \in N$ is a veto-player, then $x=$ $(a, o) \in X^{i}$ with $i \neq j$ implies $j \in C(o)$. Or, in case player $j \in N$ is a dictator, then $x=(a, o) \in X^{i}$ with $i \neq j$ implies $C(o)=\{j\}$ and $x=(a, o) \in X^{j}$ implies $C(o)=\emptyset$. These special cases can be easily extended to general voting rules as in Banks and Duggan (2000). It is common in legislative bargaining to assume that all players vote on a proposal, whereas here we assume that the proposer targets a particular winning coalition. Our methods straightforwardly extend to the case where all players vote.

Some papers in the legislative bargaining literature, like Baron and Ferejohn (1989), also consider "open rules," where the first responder to a proposal can make an amendment. Our methods can be applied to such cases, but would require the introduction of additional state variables. The same is true for papers in legislative bargaining where an accepted proposal becomes the new status quo and bargaining continues as for instance in Kalandrakis (2004b).

We now turn to the selection of the proposer. The frequently used bilateral alternating-offers procedure imposes $\rho_{2}(1, x, r)=\rho_{1}(2, x, r)=1$. Fixed rotating orders of proposers can be modeled similarly, for example the infinitely repeated order $1, \ldots, n$ is obtained by setting $\rho_{i+1}(i, x, r)=1$, where we write $i+1$ instead of $i+1 \bmod n$. Kalandrakis (2004a) and Britz et al. (2010) study the more general case where the proposer is selected by means of a Markov process. Conditional on player $i \in N$ being the current proposer, the probability that player $j \in N$ is the next proposer is given by $\bar{\rho}_{i, j}$. We then take $\rho_{j}(i, x, r)=\bar{\rho}_{i, j}$. Time-invariant selection probabilities as in Binmore (1987) are obtained as the special case in which $\bar{\rho}_{i, j}$ is independent of $i$. Finally, the rejecter-becomes-proposer protocol of Selten (1981) is obtained by setting $\rho_{r}(i, x, r)=1$.

The history at stage $k=1, \ldots, n$ of round $t \in \mathbb{N}$ is denoted by $h^{t, k}$ and the history at the end of round $t$, when nature selects the next proposer, is denoted by $h^{t}$. The initial history is given by $h^{0}=\varnothing$.

Let $t \in \mathbb{N}$. The history at stage 1 of round $t$ is given by $h^{t, 1}=\left(h^{t-1}, i^{t}\right)$. The set of all such histories is denoted by $H^{t, 1}$. The history at stage 2 of round $t$ is given by $h^{t, 2}=$ $\left(h^{t, 1}, x^{t}\right)$. The set of all such histories is denoted by $H^{t, 2}$. For $k=3, \ldots,\left|C\left(o^{t}\right)\right|+1$, in case of an acceptance in stages $2, \ldots, k-1$, the history at stage $k$ of round $t$ is given by $h^{t, k}=\left(h^{t, k-1}, 0\right)$ and the set of all such histories is denoted by $H^{t, k}$. For 
$k=3, \ldots,\left|C\left(o^{t}\right)\right|+1$, in case of an acceptance in stages $2, \ldots, k-2$ and a rejection by player $r^{t}$ in stage $k-1$, we define the history $h^{t}=\left(h^{t, k-1}, r^{t}\right)$.

A behavioral strategy $\sigma^{i}$ of player $i \in N$ is a function from the set of histories at which player $i$ has to act into a probability distribution over the set of feasible actions. The set of such behavioral strategies is denoted by $\Sigma^{i}$. The set of strategy profiles is given by $\Sigma=\times_{i \in N} \Sigma^{i}$.

Players are assumed to have von Neumann-Morgenstern utility functions. The utility for player $i \in N$ of alternative $a \in A$ is denoted by $u^{i}(a)$ and the vector of utilities across alternatives is denoted by $u^{i} \in \mathbb{R}^{A}$. The utility of the status quo is given by $u^{i}(q)$, which is normalized to be equal to 0 . We define $\bar{u}^{i}=\max _{a \in A \cup\{q\}} u^{i}(a)$ and $\underline{u}^{i}=\min _{a \in A \cup\{q\}} u^{i}(a)$. It holds that $\bar{u}^{i} \geq 0$. As is common in the literature on coalitional bargaining, we assume that $\underline{u}_{i}=0 .^{2}$

For $\sigma \in \Sigma, \pi(a, t ; \sigma, \delta) \in[0,1]$ denotes the probability of reaching agreement on alternative $a \in A$ at a round $t^{\prime} \leq t$. These probabilities are well-defined, nondecreasing in $t$, and bounded due to $\sum_{a \in A} \pi(a, t ; \sigma, \delta) \leq 1$. Hence, $\pi(a, t ; \sigma, \delta)$ converges as $t$ goes to infinity and we define $\pi(a ; \sigma, \delta)=\lim _{t \rightarrow \infty} \pi(a, t ; \sigma, \delta)$ as the limit probability. Note that $1-\sum_{a \in A} \pi(a ; \sigma, \delta)$ is equal to the probability of perpetual disagreement plus the probability that bargaining breaks down.

Given strategy profile $\sigma \in \Sigma$, we can write the expected utility of player $i \in N$ as

$$
U^{i}(\sigma, \delta)=\sum_{a \in A} \pi(a ; \sigma, \delta) u^{i}(a) .
$$

Our main analysis deals with stationary strategies, which only depend on payoffrelevant aspects of the history. Stationarity requires players to behave the same after equivalent histories. The assumption of stationarity is strong, though common in the bargaining literature. As argued in Maskin and Tirole (2001), stationary strategies prescribe the simplest form of behavior that is consistent with rationality. Moreover, without the assumption of stationarity, it follows from the results of Herrero (1985), Haller (1986), Baron and Ferejohn (1989) and Herings et al. (2017) that multilateral bargaining models lack prediction power.

At stage $(t, 1)$, it only matters which player is selected as the current proposer. At responder stages $(t, 2), \ldots,\left(t,\left|o^{t}\right|+1\right)$, only the proposer $i^{t}$ and the proposal $x^{t}$ are relevant. Formally, for every $i \in N$, for every $x=(a, o) \in X^{i}$, for every $k \in\{2, \ldots,|C(o)|+1\}$, we define

$$
\begin{aligned}
& H(i)=\left\{h \in \cup_{t \in \mathbb{N}} H^{t, 1} \mid h=\left(h^{t-1}, i\right)\right\}, \\
& H(i, x, k)=\left\{h \in \cup_{t \in \mathbb{N}} H^{t, k} \mid h=\left(h^{t-1}, i, x, 0, \ldots, 0\right)\right\} .
\end{aligned}
$$

A strategy $\sigma^{i} \in \Sigma^{i}$ of player $i \in N$ is stationary if $\sigma^{i}(h)=\sigma^{i}\left(h^{\prime}\right)$ whenever either $h, h^{\prime} \in H(i)$ or there is $j \in N \backslash\{i\}$ and $(a, o) \in X^{j}$ such that $h, h^{\prime} \in H\left(j,(a, o), k^{i}(o)\right)$.

It is convenient to define the following state space. The state where player $i \in N$ proposes is represented by $i$. The states where player $i \in N$ responds are collected in

\footnotetext{
2 Following Baron and Ferejohn (1989), it is also common in legislative bargaining to assume $\underline{u}_{i}=0$. For an analysis of the case in legislative bargaining where $\underline{u}_{i}<0$, we refer to Banks and Duggan (2006).
} 
$R^{i}=\left\{\left(j,(a, o), k^{i}(o)\right) \mid j \in N \backslash\{i\},(a, o) \in X^{j}, i \in C(o)\right\}$. We define $S^{i}=\{i\} \cup R^{i}$ as the set of all states where player $i$ is active and $S=\cup_{i \in N} S^{i}$ as the set of states where some player is active. Finally, the set $A \cup\{q\}$ contains terminal states where an alternative $a \in A$ has been accepted or the status quo $q$ results.

We represent a stationary strategy $\sigma^{i}$ of player $i \in N$ by $\sigma^{\mathrm{M}, i}=\left(\alpha^{i}, \beta^{i}\right) \in$ $\Delta\left(X^{i}\right) \times[0,1]^{R^{i}}$, where $\Delta\left(X^{i}\right)$ is the set of probability distributions on $X^{i}, \alpha^{i}(x)$ is the probability that $x \in X^{i}$ is proposed by player $i$, and, for $s=\left(j,(a, o), k^{i}(o)\right) \in R^{i}$, $\beta^{i}(s)$ is the probability that player $i$ accepts the proposal $(a, o) \in X^{j}$ made by player $j \in N \backslash\{i\}$ conditional on an acceptance by $i$ 's predecessors in the voting order $o$. A stationary strategy profile is denoted by $\sigma^{\mathrm{M}}=(\alpha, \beta)$, where $\alpha=\left(\alpha^{1}, \ldots, \alpha^{n}\right)$ and $\beta=\left(\beta^{1}, \ldots, \beta^{n}\right)$. The set of stationary strategies of player $i \in N$ is denoted by $\Sigma^{\mathrm{M}, i}$. We also define $\Sigma^{\mathrm{M},-i}=\times_{j \in N \backslash\{i\}} \Sigma^{\mathrm{M}, j}$ and $\Sigma^{\mathrm{M}}=\times_{i \in N} \Sigma^{\mathrm{M}, i}$.

A strategy profile in $\Sigma$ is a subgame perfect equilibrium (SPE) if there is no history at which some player has a profitable deviation. A subgame perfect equilibrium in stationary strategies (SSPE) is an SPE such that every player uses a stationary strategy.

\section{Quasi SSPE}

For $\delta \in[0,1)$, the existence of an SSPE in our bargaining model follows from standard results on equilibrium existence in stochastic games, see Fink (1964), Takahashi (1964) and Sobel (1971). The existence proof uses optimality conditions resulting from the one-stage deviation principle together with a system of recursive equations to pin down equilibrium utilities. We now extend this approach to $\delta \in[0,1]$.

A stationary strategy profile $\sigma^{\mathrm{M}} \in \Sigma^{\mathrm{M}}$ induces a Markov process on the state space $S \cup A \cup\{q\}$. We collect the transition probabilities in matrices $P^{S}\left(\sigma^{\mathrm{M}}, \delta\right), P^{A}\left(\sigma^{\mathrm{M}}, \delta\right)$, and $P^{q}\left(\sigma^{\mathrm{M}}, \delta\right)$ for transitions from $S$ to $S, S$ to $A$, and $S$ to $\{q\}$. We state the following result without proof.

Lemma 1 For $\delta \in[0,1]$, the stationary strategy profile $\sigma^{\mathrm{M}} \in \Sigma^{\mathrm{M}}$ induces a Markov process on $S \cup A \cup\{q\}$ with transition probabilities

$$
\Lambda\left(\sigma^{\mathrm{M}}, \delta\right)=\left[\begin{array}{ccc}
P^{S}\left(\sigma^{\mathrm{M}}, \delta\right) & P^{A}\left(\sigma^{\mathrm{M}}, \delta\right) P^{q}\left(\sigma^{\mathrm{M}}, \delta\right) \\
0 & I & 0 \\
0 & 0 & 1
\end{array}\right]
$$

All probabilities in $\Lambda\left(\sigma^{\mathrm{M}}, \delta\right)$ are continuous in $(\alpha, \beta)$ and $\delta$.

Given $\sigma^{\mathrm{M}} \in \Sigma^{\mathrm{M}}$ and $\delta \in[0,1]$, the expected utility of player $i \in N$ conditional on reaching state $s \in S$ is denoted by $v^{i}\left(s ; \sigma^{\mathrm{M}}, \delta\right)$ and the vector of utilities across states is denoted by $v^{i}\left(\sigma^{\mathrm{M}}, \delta\right) \in \mathbb{R}^{S}$. Let $\bar{\rho}^{S}=\left(\bar{\rho}^{S^{1}}, \ldots, \bar{\rho}^{S^{n}}\right) \in \mathbb{R}_{+}^{S}$ denote the initial probability distribution over states in $S$, where, for every $i \in N, \bar{\rho}^{S^{i}}=\left(\bar{\rho}_{i}, 0, \ldots, 0\right) \in$ $\mathbb{R}_{+}^{S^{i}}$. Notice that $\bar{\rho} S$ puts probability $\bar{\rho}_{i}$ on the proposer state $i \in N$ and zero probability on the responder states in $R^{i}$. The following result is given without proof. 
Lemma 2 Let $\sigma^{\mathrm{M}} \in \Sigma^{\mathrm{M}}$ and $\delta \in[0,1]$. For every $i \in N$, it holds that

$$
\begin{aligned}
v^{i}\left(\sigma^{\mathrm{M}}, \delta\right) & =\sum_{\tau=1}^{\infty} P^{S}\left(\sigma^{\mathrm{M}}, \delta\right)^{\tau-1} P^{A}\left(\sigma^{\mathrm{M}}, \delta\right) u^{i}, \\
U^{i}\left(\sigma^{\mathrm{M}}, \delta\right) & =\bar{\rho}^{S} \cdot v^{i}\left(\sigma^{\mathrm{M}}, \delta\right) .
\end{aligned}
$$

It is easily seen that at any state the profile of expected utilities across players belongs to the set $\bar{U}$ defined by

$$
\bar{U}=\operatorname{conv}\left\{\left(u^{1}(a), \ldots, u^{n}(a)\right) \in \mathbb{R}^{n} \mid a \in A \cup\{q\}\right\},
$$

where conv denotes the convex hull of a set.

Definition 3 For $\delta \in[0,1]$, the strategy profile $\sigma^{\mathrm{M}}=(\alpha, \beta) \in \Sigma^{\mathrm{M}}$ is a quasi SSPE if there exists $w \in \bar{U}^{S}$ such that, for every $i \in N$,

1. The optimality conditions hold:

$$
\begin{aligned}
& \alpha^{i} \in \arg \max _{\hat{\alpha}^{i} \in \Delta\left(X^{i}\right)} \sum_{x \in X^{i}} \hat{\alpha}^{i}(x) w^{i}(i, x, 2), \\
& \beta^{i}(s) \in \arg \max _{\hat{\beta}^{i} \in[0,1]} \hat{\beta}^{i} w^{i}\left(j,(a, o), k^{i}(o)+1\right) \\
& \quad+\left(1-\hat{\beta}^{i}\right) \sum_{i^{\prime} \in N} \delta \rho_{i^{\prime}}(j,(a, o), i) w^{i}\left(i^{\prime}\right), \quad s=\left(j,(a, o), k^{i}(o)\right) \in R^{i},
\end{aligned}
$$

where $w^{i}\left(j,(a, o), k^{i}(o)+1\right)=u^{i}(a)$ if $i$ is the last responder in $o$.

2. The system of recursive equations holds:

$$
w^{i}=P^{A}\left(\sigma^{\mathrm{M}}, \delta\right) u^{i}+P^{S}\left(\sigma^{\mathrm{M}}, \delta\right) w^{i}
$$

The following result establishes the existence of a quasi SSPE. We defer all proofs to the "Appendix".

Theorem 4 For every $\delta \in[0,1]$, a quasi SSPE exists.

The next result states that for $\delta \in[0,1)$, a quasi SSPE is an SSPE and vice versa. This result is well-known and therefore stated without proof. It also follows as a consequence of Theorem 15 in this paper.

Theorem 5 For every $\delta \in[0,1)$, the set of SSPEs coincides with the set of quasi SSPES.

\section{Two motivating examples}

In this section, we present two examples, which reveal that a quasi SSPE may fail to be an SSPE when $\delta=1$.

The first example considers the simplest imaginable set-up. Two players bargain about the implementation of an alternative from which both benefit. An SSPE exists for all values of $\delta$, but at $\delta=1$ there is a quasi SSPE that fails to be an SSPE. 
Example 6 Common-interest alternating-offers bargaining

Consider alternating-offers bargaining with $N=\{1,2\}$, set of alternatives $A=\{\hat{a}\}$, and, for $i \in N$ and $j \in N \backslash\{i\}$, set of feasible proposals $X^{i}=\{(\hat{a},(j))\}$. For each player, the utility of $\hat{a}$ is 1 and the utility of $q$ is 0 . This example is a special case of Muthoo (1991).

To simplify the exposition, we restrict ourselves to symmetric stationary strategies. Player $i \in N$ proposes $(\hat{a},(j))$ with probability 1 and accepts $\hat{a}$ with probability $\beta \in[0,1]$. Conditional on being the proposer, the expected utility of a player is denoted by $v^{\mathrm{p}}=v^{i}(i ; \beta, \delta)$. Conditional on being the responder, the expected utility of a player is denoted by $v^{\mathrm{r}}=v^{j}(i, x, 2 ; \beta, \delta)$.

According to Definition 3 of a quasi SSPE, we have to find $\beta, w^{\mathrm{p}}, w^{\mathrm{r}} \in[0,1]$ such that

$$
\begin{aligned}
& \beta \in \arg \max _{\hat{\beta} \in[0,1]} \hat{\beta}+(1-\hat{\beta}) \delta w^{\mathrm{p}}, \\
& w^{\mathrm{p}}=\beta+(1-\beta) \delta w^{\mathrm{r}} \\
& w^{\mathrm{r}}=\beta+(1-\beta) \delta w^{\mathrm{p}} .
\end{aligned}
$$

In case $\delta \in[0,1)$, we obtain the unique solution $\beta=1$ and $w^{\mathrm{p}}=w^{\mathrm{r}}=1$. This solution yields an SSPE by Theorem 5. In this SSPE, the proposal $(\hat{a},(j))$ is accepted with probability 1 , so $v^{\mathrm{p}}=v^{\mathrm{r}}=1$.

We now turn to the case $\delta=1$. Any $\beta \in[0,1]$ together with $w^{\mathrm{p}}=w^{\mathrm{r}}=1$ is a quasi SSPE. Any solution with $\beta>0$ is a quasi SSPE that does correspond to an SSPE. In such a solution, the proposal $(\hat{a},(j))$ is accepted with probability $\beta>0$ in each bargaining round, so ultimately there is acceptance of the proposal with probability one. The solution $\beta=0$ is problematic, since it leads to perpetual disagreement, so $v^{\mathrm{p}}=v^{\mathrm{r}}=0$, which is not in accordance with the solution $w^{\mathrm{p}}=w^{\mathrm{r}}=1$. Moreover, when $\beta=0$, a responder has a profitable one-stage deviation to acceptance. The solution $\beta=0$ is a quasi SSPE, but does not correspond to an SSPE.

The following example presents a case without an SSPE at $\delta=1$, even though a quasi SSPE does exist when $\delta=1$. It involves coalitional bargaining with three players, where utilities display the features of the Condorcet paradox.

\section{Example 7 Hedonic coalition formation}

Consider a game of hedonic coalition formation with $N=\{1,2,3\}$. There are three possible alternatives $A=\left\{a^{12}, a^{23}, a^{31}\right\}$ corresponding to each of the three possible two-player coalitions that may form. Utilities are given by $u\left(a^{12}\right)=(2,1,0)$, $u\left(a^{23}\right)=(0,2,1)$, and $u\left(a^{31}\right)=(1,0,2)$. Each player can choose between two coalitions to form, so $X^{1}=\left\{\left(a^{12},(2)\right),\left(a^{31},(3)\right)\right\}, X^{2}=\left\{\left(a^{23},(3)\right),\left(a^{12},(1)\right)\right\}$, and $X^{3}=\left\{\left(a^{31},(1)\right),\left(a^{23},(2)\right)\right\}$. The utilities display a cyclical pattern that resembles the Condorcet paradox in the sense that players 2 and 3 prefer coalition $\{2,3\}$ to $\{1,2\}$, players 3 and 1 prefer coalition $\{3,1\}$ to $\{2,3\}$, and players 1 and 2 prefer coalition $\{1,2\}$ to $\{3,1\}$.

We consider the rejecter-becomes-proposer protocol as introduced in Selten (1981). The proposer in round 1 is selected randomly. A player $i \in N$ proposes either $x^{+}=$ $\left(a^{i, i+1},(i+1)\right)$ or $x^{-}=\left(a^{i-1, i},(i-1)\right)$, where we write $i+1$ or $i-1$ instead of 
$i+1 \bmod 3$, respectively $i-1 \bmod 3$. A proposer $i$ prefers $x^{+}$to $x^{-}$. If the responder, say player $j$, accepts, then negotiations end with $i$ and $j$ forming a coalition. Otherwise, $j$ is next in turn to make a proposal, unless breakdown occurs.

To simplify the exposition, we restrict ourselves to symmetric stationary strategies. Such strategies are summarized by three probabilities, $\alpha, \beta^{-}$, and $\beta^{+}$, where $\alpha$ denotes the probability of proposing $x^{+}, \beta^{-}$is the probability by which a player accepts his less preferred coalition, and $\beta^{+}$is the probability by which a player accepts his most preferred coalition. We use $v=v^{i}\left(i ;\left(\alpha, \beta^{-}, \beta^{+}\right), \delta\right)$ to denote the expected utility for proposer $i, v^{+}=v^{i+1}\left(i ;\left(\alpha, \beta^{-}, \beta^{+}\right), \delta\right)$ to denote the expected utility of $i$ 's most preferred partner, and $v^{-}=v^{i-1}\left(i ;\left(\alpha, \beta^{-}, \beta^{+}\right), \delta\right)$ to denote the expected utility of $i$ 's least preferred partner in proposer state $i$.

Proposition 8 extends the non-existence result for pure strategies in Bloch (1996) at $\delta=1$ to mixed strategies. It also extends the non-existence result for pure strategies in Livshits (2002) at $\delta=0.99$ to all $\delta \in\left(\frac{1}{2}, 1\right]$.

Proposition 8 1. For $\delta \in[0,1 / 2]$, the unique symmetric SSPE is given by $\left(\alpha, \beta^{-}, \beta^{+}\right)=(1,1,1)$ with utilities $v=2, v^{+}=1$, and $v^{-}=0$.

2. For $\delta \in(1 / 2,1)$, the unique symmetric SSPE is given by

$$
\alpha=1, \beta^{-}=\frac{-\left(1-\delta^{2}\right)+\sqrt{(1-\delta)(1+2 \delta)}}{\delta^{2}} \in(0,1), \text { and } \beta^{+}=1
$$

with utilities

$$
v=1 / \delta \in(1,2), v^{+}=1, \text { and } v^{-}=\frac{1-\sqrt{(1-\delta)(1+2 \delta)}}{\delta} \in(0,1) .
$$

3. For $\delta=1$, the unique symmetric quasi SSPE is given by $\left(\alpha, \beta^{-}, \beta^{+}\right)=(1,0,1)$ with values $w=w^{+}=w^{-}=1$. A symmetric SSPE does not exist.

Consider an SSPE. For $\delta \in[0,1)$, the proposer always selects his most preferred coalition, i.e., $x^{+}$. A responder always accepts when the proposal involves his most preferred coalition, i.e., $\beta^{+}=1$.

For $\delta \leq 1 / 2$, a player also always accepts his less preferred coalition, i.e., $\beta^{-}=1$, so there is agreement with probability 1 on the proposer's most preferred coalition.

For $\delta \in(1 / 2,1)$, a player randomizes when responding to his less preferred coalition, i.e., $\beta^{-} \in(0,1)$. Negotiations end in round $t$ with probability $(1-$ $\left.\beta^{-}\right)^{t-1} \delta^{t-1} \beta^{-}>0$. Perpetual disagreement occurs with probability zero. As long as there is no agreement, the equilibrium path exhibits the following cyclical behavior: First proposer $i$ proposes to $i+1$, who in turn proposes to $i-1$, who in turn proposes to $i$, and so on. Since $\delta<1$ means that delay is costly, the SSPE is Pareto inefficient. When $\delta$ goes to $1, v+v^{+}+v^{-}$goes to 3 with a slope converging to $+\infty$. In Fig. 1 we plot $v+v^{+}+v^{-}$and we observe an U-shape on the domain $(1 / 2,1)$, with a minimum around 2.60 at $\delta \approx 0.81$.

At $\delta=1$, there is a unique symmetric quasi SSPE. The proposer always selects his most preferred coalition, i.e., $x^{+}$, but such a proposal is rejected with probability 1 by the responder. The players end up in perpetual disagreement with probability 1 and 


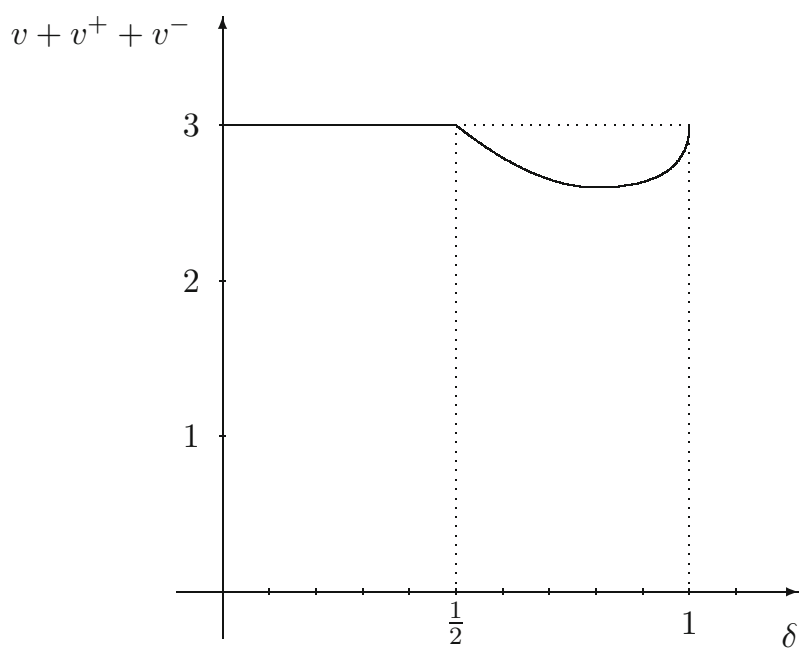

Fig. 1 The sum of utilities as a function of $\delta$ in Example 7

utilities $v=v^{+}=v^{-}=0$, which are different from the values in the quasi SSPE, where $w=w^{+}=w^{-}=1$. This quasi SSPE fails to be an SSPE at $\delta=1$, since the proposer has a profitable one-stage deviation by proposing $x^{-}$to form a coalition with his less preferred coalition partner.

\section{The system of recursive equations}

A common feature of both Examples 6 and 7 is that the system of recursive equations (5) for a player $i \in N$ may have a solution $w^{i}$ which is different from $v^{i}\left(\sigma^{\mathrm{M}}, \delta\right)$. In this section, we analyze the system of recursive equations in detail and we present necessary and sufficient conditions to guarantee that a solution $w^{i}$ coincides with $v^{i}\left(\sigma^{\mathrm{M}}, \delta\right)$. The next proposition establishes that $v^{i}\left(\sigma^{\mathrm{M}}, \delta\right)$ is always a solution to the system of recursive equations.

Proposition 9 Let $\sigma^{\mathrm{M}} \in \Sigma^{\mathrm{M}}$ and $\delta \in[0,1]$. For every $i \in N$, it holds that $v^{i}\left(\sigma^{\mathrm{M}}, \delta\right)$ is a solution to the system of recursive equations (5).

If the system of recursive equations (5) has a unique solution, then it must be equal to $v^{i}\left(\sigma^{\mathrm{M}}, \delta\right)$ by virtue of Proposition 9 . The system of recursive equations can be rewritten as $\left[I-P^{S}\left(\sigma^{\mathrm{M}}, \delta\right)\right] w^{i}=P^{A}\left(\sigma^{\mathrm{M}}, \delta\right) u^{i}$ and these equations admit a unique solution if and only if the matrix $I-P^{S}\left(\sigma^{\mathrm{M}}, \delta\right)$ is non-singular. In that case, we have $w^{i}=\left[I-P^{S}\left(\sigma^{\mathrm{M}}, \delta\right)\right]^{-1} P^{A}\left(\sigma^{\mathrm{M}}, \delta\right) u^{i}$. The matrix $I-P^{S}\left(\sigma^{\mathrm{M}}, \delta\right)$ is non-singular if and only if the matrix $P^{S}\left(\sigma^{\mathrm{M}}, \delta\right)$ does not have an eigenvalue equal to 1 .

To obtain conditions such that $I-P^{S}\left(\sigma^{\mathrm{M}}, \delta\right)$ is non-singular, we follow the approach in Solow (1952). A matrix $M \in \mathbb{R}_{+}^{S \times S}$ is said to be irreducible if there 
does not exist a permutation matrix $\Pi \in \mathbb{R}^{S \times S}$ such that

$$
\Pi M \Pi^{-1}=\left[\begin{array}{cc}
\tilde{M}_{11} & \tilde{M}_{12} \\
0 & \tilde{M}_{22}
\end{array}\right],
$$

where $\tilde{M}_{11}$ and $\tilde{M}_{22}$ are square matrices. A $1 \times 1$ matrix is irreducible by definition, also if its unique element is equal to zero. Often it is possible to further decompose $\tilde{M}_{11}$ or $\tilde{M}_{22}$. A finest decomposition $\Pi M \Pi^{-1}$ of $M$ consists of an upper-triangular block form whose diagonal blocks, denoted by $\tilde{M}_{11}, \ldots, \tilde{M}_{f f}$, are irreducible. For $e=1, \ldots, f$, let $S_{e}$ be the set of states associated with $\tilde{M}_{e e}$. It follows from Solow (1952) that a finest decomposition of $M$ always exists and that all finest decompositions result in the same collection $\left\{S_{e} \mid e=1, \ldots, f\right\}$.

Let $\tilde{P}\left(\sigma^{\mathrm{M}}, \delta\right)$ be a finest decomposition of $P^{S}\left(\sigma^{\mathrm{M}}, \delta\right)$. Then we can write

$$
\tilde{P}\left(\sigma^{\mathrm{M}}, \delta\right)=\left[\begin{array}{cccc}
\tilde{P}_{11}\left(\sigma^{\mathrm{M}}, \delta\right) & \tilde{P}_{12}\left(\sigma^{\mathrm{M}}, \delta\right) & \cdots & \tilde{P}_{1 f}\left(\sigma^{\mathrm{M}}, \delta\right) \\
0 & \tilde{P}_{22}\left(\sigma^{\mathrm{M}}, \delta\right) & \cdots & \tilde{P}_{2 f}\left(\sigma^{\mathrm{M}}, \delta\right) \\
\vdots & \vdots & \ddots & \vdots \\
0 & 0 & \cdots & \tilde{P}_{f f}\left(\sigma^{\mathrm{M}}, \delta\right)
\end{array}\right],
$$

where, for $e=1, \ldots, f$, the diagonal block $\tilde{P}_{e e}\left(\sigma^{\mathrm{M}}, \delta\right)$ is an irreducible square matrix. We denote the states associated with $\tilde{P}_{e e}\left(\sigma^{\mathrm{M}}, \delta\right)$ by $S_{e}\left(\sigma^{\mathrm{M}}, \delta\right)$.

The following result gives necessary and sufficient conditions such that the matrix $I-P^{S}\left(\sigma^{\mathrm{M}}, \delta\right)$ is non-singular.

Proposition 10 The system of recursive equations (5) has a unique solution if and only if, for every $e=1, \ldots, f$, the irreducible block $\tilde{P}_{e e}\left(\sigma^{\mathrm{M}}, \delta\right)$ in a finest decomposition $\tilde{P}\left(\sigma^{\mathrm{M}}, \delta\right)$ of $P^{S}\left(\sigma^{\mathrm{M}}, \delta\right)$ has at least one row sum less than 1 .

Consider the set of states $S_{e}\left(\sigma^{\mathrm{M}}, \delta\right)$ for some $e=1, \ldots, f$. The matrix $\tilde{P}_{e e}\left(\sigma^{\mathrm{M}}, \delta\right)$ describes the transition probabilities on the subset $S_{e}\left(\sigma^{\mathrm{M}}, \delta\right)$. Because $\tilde{P}_{e e}\left(\sigma^{\mathrm{M}}, \delta\right)$ is irreducible, each state in $S_{e}\left(\sigma^{\mathrm{M}}, \delta\right)$ is reached with positive probability after a finite number of steps from any other state in $S_{e}\left(\sigma^{\mathrm{M}}, \delta\right)$. In case a state is reached which corresponds to a row sum less than 1 , then with positive probability a transition to a state outside $S_{e}\left(\sigma^{\mathrm{M}}, \delta\right)$ occurs, being either an absorbing state in $A \cup\{q\}$ or a state in $S_{e^{\prime}}\left(\sigma^{\mathrm{M}}, \delta\right)$ with $e^{\prime}>e$. In both cases, the Markov process never returns to states in $S_{e}\left(\sigma^{\mathrm{M}}, \delta\right)$. Repeating this logic a finite number of times leads to the conclusion that for any initial state in $S$ we eventually reach an absorbing state in $A \cup\{q\}$ with probability 1 .

Proposition 11 Let $\sigma^{\mathrm{M}}=(\alpha, \beta) \in \Sigma^{\mathrm{M}}$ and $\delta \in[0,1]$. If $\delta<1$ or $[\delta=1$ and for every $i \in N$, there is $x=(a, o) \in X^{i}$ such that $\alpha^{i}(x)>0$, and, for every $j \in C(o)$, $\left.\beta^{j}\left(i, x, k^{j}(o)\right)>0\right]$, then the system of recursive equations (5) has a unique solution.

According to Proposition 11, the system of recursive equations (5) is guaranteed to have $v^{i}\left(\sigma^{\mathrm{M}}, \delta\right)$ as the unique solution in case of costly delay. We also obtain a unique solution in the case of costless delay when every player makes with positive probability 
a proposal that is accepted with positive probability by every responder. Proposition 11 can be applied to Example 6 to conclude that, for every $i \in N, w^{i}=v^{i}(\beta, \delta)$ whenever $\delta<1$ or $\beta>0$. It can be applied to Example 7 to conclude that, for every $i \in N$, $w^{i}=v^{i}(\alpha, \beta, \delta)$ whenever $\delta<1$ or $\left[\delta=1, \alpha>0\right.$, and $\left.\beta^{-}>0\right]$ or $[\delta=1, \alpha<1$, and $\left.\beta^{+}>0\right]$.

Many papers in the bargaining literature either assume that every player $i \in N$ is selected to be the proposer with a time-invariant probability $\bar{\rho}_{i}>0$ or assume that there is a fixed rotating order among all players. We call a bargaining protocol irreducible if, for every $i \in N$, for every $x^{i}=\left(a^{i}, o^{i}\right) \in X^{i}$, for every $r^{i} \in C(o)$, the matrix

$$
\left[\begin{array}{ccc}
\rho_{1}\left(1, x^{1}, r^{1}\right) & \cdots & \rho_{1}\left(n, x^{n}, r^{n}\right) \\
\vdots & & \vdots \\
\rho_{n}\left(1, x^{1}, r^{1}\right) & \cdots & \rho_{n}\left(n, x^{n}, r^{n}\right)
\end{array}\right] .
$$

is irreducible. The bargaining protocols with positive time-invariant probabilities and the fixed rotating order among all players are two examples of an irreducible bargaining protocol.

Proposition 12 Let the bargaining protocol be irreducible, $\sigma^{\mathrm{M}}=(\alpha, \beta) \in \Sigma^{\mathrm{M}}$, and $\delta \in[0,1]$. The system of recursive equations (5) has a unique solution if and only if $\delta<1$ or $\left[\delta=1\right.$ and there is $i^{\prime} \in N$ and $x^{\prime}=\left(a^{\prime}, o^{\prime}\right) \in X^{i^{\prime}}$ such that $\alpha^{i^{\prime}}\left(x^{\prime}\right)>0$ and, for every $\left.j \in C\left(o^{\prime}\right), \beta^{j}\left(i^{\prime}, x^{\prime}, k^{j}\left(o^{\prime}\right)\right)>0\right]$.

Both Examples 6 and 7 feature irreducible bargaining protocols, so Proposition 12 can be applied to both of them. When $\delta=1$ and $\beta=0$ in Example 6 or $\delta=$ 1 and $\left(\alpha, \beta^{-}, \beta^{+}\right)=(1,0,1)$ in Example 7, the conditions in Proposition 12 are violated. It follows that the system of recursive equations (5) has infinitely many solutions and there is no guarantee that a solution $w^{i}$ corresponds to $v^{i}\left(\sigma^{\mathrm{M}}, \delta\right)$. Notice that the uniqueness of $w^{\mathrm{p}}=w^{\mathrm{r}}=1$ in Example 6 and $w=w^{+}=w^{-}=1$ in Example 7 means that the combination of the optimality conditions and the system of recursive equations has a unique solution, but does not imply that the system of recursive equations by itself has a unique solution.

We conclude this section with an analysis of the general case, where the system of recursive equations (5) is allowed to be singular. It now follows from Proposition 10 that there is at least one irreducible block $\tilde{P}_{e e}\left(\sigma^{\mathrm{M}}, \delta\right)$ in a finest decomposition of $P^{S}\left(\sigma^{\mathrm{M}}, \delta\right)$ with all row sums in this block equal to one. Once a state in the set $S_{e}\left(\sigma^{\mathrm{M}}, \delta\right)$ is reached, the Markov process never leaves it, so these states lead to perpetual disagreement. It follows immediately that such a situation can only occur if $\delta=1$.

For $\sigma^{\mathrm{M}} \in \Sigma^{\mathrm{M}}$ and $\delta \in[0,1]$, the set of all states leading to perpetual disagreement is denoted by $S^{\mathrm{pd}}\left(\sigma^{\mathrm{M}}, \delta\right)$. Notice that $S^{\mathrm{pd}}\left(\sigma^{\mathrm{M}}, \delta\right)=\emptyset$ if $\delta<1$. We have that, for every $s \in S^{\mathrm{pd}}\left(\sigma^{\mathrm{M}}, \delta\right)$, for every $i \in N, v^{i}\left(s ; \sigma^{\mathrm{M}}, \delta\right)=0$. The next proposition uses this fact to give an easy way to check whether a solution to the system of recursive equations (5) is equal to $v^{i}\left(\sigma^{\mathrm{M}}, \delta\right)$ in the general case. 
Proposition 13 Let $\sigma^{\mathrm{M}} \in \Sigma^{\mathrm{M}}$ and $\delta \in[0,1]$. For $i \in N$, let $w^{i}$ be a solution to the system of recursive equations (5). It holds that $w^{i}=v^{i}\left(\sigma^{\mathrm{M}}, \delta\right)$ if and only if, for every $s \in S^{\mathrm{pd}}\left(\sigma^{\mathrm{M}}, \delta\right), w^{i}(s)=0$.

We can use Proposition 13 in Example 6 when $\delta=1$ and $\beta=0$. Since every state $s \in S$ leads to perpetual disagreement, the solution $w^{\mathrm{p}}=w^{\mathrm{r}}=1$ does not correspond to the actual utilities generated by the strategy profile. For the strategy profile $\left(\alpha, \beta^{-}, \beta^{+}\right)=(1,0,1)$ in Example 7 , all the proposer states lead to perpetual disagreement when $\delta=1$. The solution $\left(w, w^{+}, w^{-}\right)=(1,1,1)$ is therefore not in accordance with the actual utilities induced by the strategy profile. In this example, not all states lead to perpetual disagreement. In a responder state where the proposal corresponds to the most preferred coalition of a player, immediate acceptance takes place with probability 1 .

\section{Stationary best responses}

In this section, we present a full characterization of the stationary best response of a player to any stationary strategy profile. A stationary best response is shown to always exist.

Consider a player $i \in N$. If all other players use a stationary strategy, then player $i$ has to solve a Markov Decision Problem in order to find a stationary best response. The next proposition states that player $i$ always has a stationary best response and provides a characterization.

Proposition 14 Let $i \in N, \sigma^{\mathrm{M},-i} \in \Sigma^{\mathrm{M},-i}$, and $\delta \in[0,1]$. There exists a stationary best response $\sigma^{\mathrm{M}, i}$ for player $i$ against $\sigma^{\mathrm{M},-i}$. Moreover, $\sigma^{\mathrm{M}, i}$ is a stationary best response if and only if there exists $w^{i} \in \mathbb{R}_{+}^{S}$ such that

1. The optimality conditions (4) hold.

2. The system of recursive equations (5) holds.

3. For every $s \in S^{\mathrm{pd}}\left(\sigma^{\mathrm{M}}, \delta\right), w^{i}(s)=0$.

Player $i \in N$ has a stationary best response to any stationary strategy profile, also when delay is costless. The characterization imposes the optimality conditions as well as the system of recursive equations and requires $w^{i}(s)=0$ whenever $s \in S$ is a state leading to perpetual disagreement. We conclude from Proposition 13 that $w^{i}=v^{i}\left(\sigma^{\mathrm{M}}, \delta\right)$. For $\delta \in[0,1)$, it holds that $S^{\mathrm{pd}}\left(\sigma^{\mathrm{M}}, \delta\right)=\emptyset$, so player $i$ 's best responses are fully characterized by the optimality conditions and the system of recursive equations.

We now return to Example 6, where we make the player explicit in the notation for a strategy. Consider the problematic case where $\delta=1$ and $\beta^{2}=0$, so player 2 always rejects the proposal. Then we obtain the following optimality conditions and system of recursive equations for player 1 :

$$
\begin{aligned}
& \beta^{1} \in \arg \max _{\hat{\beta}^{1}} \hat{\beta}^{1}+\left(1-\hat{\beta}^{1}\right) w^{\mathrm{p}}, \\
& w^{\mathrm{p}}=w^{\mathrm{r}}, \\
& w^{\mathrm{r}}=\beta^{1}+\left(1-\beta^{1}\right) w^{\mathrm{p}} .
\end{aligned}
$$


There are two types of solutions to this system. The first type of solution has $0<$ $\beta^{1} \leq 1$ and $w^{\mathrm{p}}=w^{\mathrm{r}}=1$. Since there are no states with perpetual disagreement, we conclude from Proposition 14 that any of these solutions corresponds to a stationary best response for player 1 . The second type of solution has $\beta^{1}=0$ and $w^{\mathrm{p}}=w^{\mathrm{r}} \geq 1$. Now the third condition of Proposition 14 comes into play. Since both the proposer and the responder state lead to perpetual disagreement and belong to $S^{\mathrm{pd}}\left(\sigma^{\mathrm{M}}, \delta\right)$, the fact that $w^{\mathrm{p}}=w^{\mathrm{r}} \neq 0$ leads to the conclusion that none of these solutions corresponds to a stationary best response for player 1 .

Next consider Example 7 in the problematic case where $\delta=1$ and $\left(\alpha^{2}, \beta^{2,-}, \beta^{2,+}\right)=$ $\left(\alpha^{3}, \beta^{3,-}, \beta^{3,+}\right)=(1,0,1)$, so player 2 always proposes to form a coalition with player 3 , player 3 always proposes to form a coalition with player 1, player 2 always rejects a coalition with player 1 and accepts a coalition with player 3 , and player 3 always rejects a coalition with player 2 and accepts a coalition with player 1 . We use $w^{1}, w^{1,-}$, and $w^{1,+}$ to denote $w^{1}(1), w^{1}\left(3,\left(a^{31},(1)\right), 2\right)$, and $w^{1}\left(2,\left(a^{12},(1)\right), 2\right)$, the values at states where player 1 proposes, responds to the less preferred coalition with player 3 , and responds to the most preferred coalition with player 2 , respectively. We obtain the following optimality conditions and system of recursive equations for player 1:

$$
\begin{aligned}
& \alpha^{1} \in \arg \max _{\hat{\alpha}^{1} \in[0,1]} \hat{\alpha}^{1} w^{1,-}+\left(1-\hat{\alpha}^{1}\right), \\
& \beta^{1,-} \in \arg \max _{\hat{\beta}^{1,-} \in[0,1]} \hat{\beta}^{1,-}+\left(1-\hat{\beta}^{1,-}\right) w^{1}, \\
& \beta^{1,+} \in \arg \max _{\hat{\beta}^{1,+} \in[0,1]} 2 \hat{\beta}^{1,+}+\left(1-\hat{\beta}^{1,+}\right) w^{1,-}, \\
& w^{1}=\alpha^{1} w^{1,-}+\left(1-\alpha^{1}\right), \\
& w^{1,-}=\beta^{1,-}+\left(1-\beta^{1,-}\right) w^{1}, \\
& w^{1,+}=2 \beta^{1,+}+\left(1-\beta^{1,+}\right) w^{1,-} .
\end{aligned}
$$

To obtain the first condition, notice that if player 1 proposes to form a coalition with player 2, this proposal is rejected. It is followed by a proposal from player 2 to form a coalition with player 3 that is rejected as well, and next a proposal by player 3 to form a coalition with player 1 , which has a value $w^{1,-}$ for player 1 . If instead, player 1 proposes to form a coalition with player 3 , then such a proposal is accepted and gives utility 1 to player 1 . The reasoning behind the other conditions is analogous.

There are two types of solutions to this system. The first type of solution has $\alpha^{1}\left(1-\beta^{1,-}\right)<1, \beta^{1,+}=1, w^{1}=w^{1,-}=1$, and $w^{1,+}=2$. Player 1 either proposes with positive probability to form a coalition with player 3 , a proposal that is accepted for sure and gives a utility of 1 , or proposes for sure to player 2 , a proposal that is rejected by player 2, and is followed by a proposal by player 3 to form a coalition with player 1 , a proposal that is accepted with positive probability. This type of solution ultimately results in the formation of coalition $\{1,3\}$ with probability 1 . In the counterfactual case where player 2 proposes to player 1, player 1 would accept such a proposal for sure, leading to a utility of 2 for player 1 . Since there are no states with perpetual disagreement, we can use Proposition 14 to conclude that any such solution yields a stationary best response for player 1 .

The second type of solution has $\alpha^{1}=1$ and $\beta^{1,-}=0$. Moreover, it holds that $w^{1}=w^{1,-} \geq 1$. There are three subcases. If $w^{1} \in[1,2)$, then it holds that $\beta^{1,+}=1$ 
and $w^{1,+}=2$. If $w^{1}=2$, then it holds that $\beta^{1,+} \in[0,1]$ and $w^{1,+}=2$. If $w^{1}>2$, then it holds that $\beta^{1,+}=0$ and $w^{1,+}=w^{1}$. In any of these these solutions, the state where player 1 is the proposer leads to perpetual disagreement. Since $w^{1} \geq 1$, the third condition of Proposition 14 is violated.

\section{Stationary subgame perfect equilibrium}

The definition of SSPE states that each player's strategy is a stationary best response to the stationary strategies of the other players. The following proposition therefore follows trivially from Proposition 14.

Theorem 15 Let $\delta \in[0,1]$. The strategy profile $\sigma^{\mathrm{M}} \in \Sigma^{\mathrm{M}}$ is an SSPE if and only if, for every $i \in N$, there exists $w^{i} \in \mathbb{R}_{+}^{S}$ such that

\section{The optimality conditions (4) hold.}

2. The system of recursive equations (5) holds.

3. For every $s \in S^{\mathrm{pd}}\left(\sigma^{\mathrm{M}}, \delta\right), w^{i}(s)=0$.

In case $\delta<1$, the set of states $S^{\mathrm{pd}}\left(\sigma^{\mathrm{M}}, \delta\right)$ leading to perpetual disagreement is empty and it is sufficient to restrict attention to the first two conditions of Theorem 15. Since these two conditions correspond to the definition of a quasi SSPE, we find that the set of quasi SSPEs coincides with the set of SSPEs. More generally, a quasi SSPE such that no state leads to perpetual disagreement is an SSPE.

By Theorem 4, a quasi SSPE exists, i.e., there is a strategy profile $\sigma^{\mathrm{M}} \in \Sigma^{\mathrm{M}}$ such that, for every $i \in N$, Conditions 1 and 2 of Theorem 15 hold. By Proposition 14, for every $i \in N$, a stationary best response always exists, i.e., fixing $\sigma^{\mathrm{M},-i} \in \Sigma^{\mathrm{M},-i}$, there is always a solution to Conditions 1,2 , and 3 of Theorem 15. It is therefore somewhat surprising that an SSPE, i.e., a strategy profile $\sigma^{\mathrm{M}} \in \Sigma^{\mathrm{M}}$ such that, for every $i \in N$, Conditions 1, 2, and 3 of Theorem 15 hold, may not exist. The reason is that although Conditions 1 and 2 of Theorem 15 are continuous in $\sigma^{\mathrm{M}}$ and $\delta$, Condition 3 is not. A sequence of strategy profiles such that there is no perpetual disagreement at any state may converge to a strategy profile with perpetual disagreement at some state, so at the limit an additional condition, Condition 3 of Theorem 15, has to be satisfied.

Alós-Ferrer and Ritzberger (2017) show for perfect information games that there is an equivalence between the one-stage deviation principle and subgame perfect equilibrium when preferences are lower semi-continuous. Theorem 15 obtains an equivalence between the one-stage deviation principle and SSPE for our class of bargaining models. Due to the chance moves, the bargaining models studied in this paper are not part of the class of perfect information games. Our utility functions are lower semi-continuous, both when defined in terms of plays and when defined in terms of stationary strategies. Conditions 2 and 3 of Theorem 15 guarantee that the values $w$ are equal to the utilities generated by the strategy profile $\sigma^{\mathrm{M}}$. Condition 1 of Theorem 15 then corresponds to the one-stage deviation principle. The use of stationary strategies has the advantage that Condition 1 only involves finitely many optimization problems.

Following the approach of Binmore et al. (1986), it is common practice in bargaining theory to study the limit of SSPEs when $\delta$ goes to one. Since the set $\Sigma^{\mathrm{M}}$ is compact, 
any sequence of SSPE strategy profiles has a convergent subsequence. We refer to a limit of SSPE strategy profiles when $\delta$ goes to one as a limit SSPE. The following results are immediate.

Corollary 16 If $\sigma^{\mathrm{M}} \in \Sigma^{\mathrm{M}}$ is a limit SSPE such that $S^{\mathrm{pd}}\left(\sigma^{\mathrm{M}}, 1\right)=\emptyset$, then $\sigma^{\mathrm{M}}$ is an SSPE at $\delta=1$.

Corollary 17 If there is no SSPE at $\delta=1$, then for every limit SSPE $\sigma^{\mathrm{M}}$ it holds that $S^{\mathrm{pd}}\left(\sigma^{\mathrm{M}}, 1\right) \neq \emptyset$.

Herings and Houba (2016) study a model of legislative bargaining with costless delay where proposers are selected according to a time-invariant probability distribution. There are three alternatives with utilities giving rise to a Condorcet paradox as in Example 7. The relevant parameters of the model are the time-invariant selection probabilities and the utilities of the alternatives. It is shown that the parameter space is partitioned in two sets of positive Lebesgue measure, one for which a generically unique SSPE exists and one where non-existence prevails. Corollary 17 implies that, for the latter class, limit SSPEs will exhibit perpetual disagreement.

There are several general existence results that establish non-emptiness and uppersemi continuity of the set of SSPEs in bargaining models, see Banks and Duggan (2000, 2006) and Duggan (2017). In these references, the set of alternatives is a continuum and the utility functions are continuous. The bargaining procedure is less general than in our model. Our results are therefore complementary.

\section{Concluding remarks}

Our main results show that the equilibrium analysis of strategic bargaining models under costless delay exhibits important subtleties. The standard characterization of an SSPE under costly delay consists of optimality conditions corresponding to the one-stage deviation principle together with a system of recursive equations to pin down equilibrium utilities. This characterization provides necessary but not sufficient conditions in case delay is costless. A strategy profile that satisfies the conditions in this characterization is therefore called a quasi SSPE.

The paper shows that a quasi SSPE always exists, but that a quasi SSPE need not be an SSPE. Problems occur when the system of recursive equations is singular, which is the case if and only if the strategy profile does not exclude perpetual disagreement in some states. Furthermore, care should be taken when one resorts to limits of SSPEs under vanishing costs of delay. In our motivating example of hedonic coalition formation, the limit is unique, but does not yield an SSPE under costless delay and, in fact, under costless delay there is no SSPE at all. We present a characterization of an SSPE that is valid for the case of costly as well as costless delay.

Open Access This article is licensed under a Creative Commons Attribution 4.0 International License, which permits use, sharing, adaptation, distribution and reproduction in any medium or format, as long as you give appropriate credit to the original author(s) and the source, provide a link to the Creative Commons licence, and indicate if changes were made. The images or other third party material in this article are included 
in the article's Creative Commons licence, unless indicated otherwise in a credit line to the material. If material is not included in the article's Creative Commons licence and your intended use is not permitted by statutory regulation or exceeds the permitted use, you will need to obtain permission directly from the copyright holder. To view a copy of this licence, visit http://creativecommons.org/licenses/by/4.0/.

\section{Appendix: Proofs}

\section{Proof of Theorem 4.}

For every $i \in N$, define the function $f^{i}: \Sigma^{\mathrm{M}} \times \bar{U}^{S} \rightarrow\left[0, \bar{u}^{i}\right]^{S}$ by $f^{i}\left(\sigma^{\mathrm{M}}, w\right)=$ $P^{A}\left(\sigma^{\mathrm{M}}, \delta\right) u^{i}+P^{S}\left(\sigma^{\mathrm{M}}, \delta\right) w^{i}$. By Lemma 1 , the function $f^{i}$ is continuous.

Since $f^{i}\left(\sigma^{\mathrm{M}}, w\right)=P^{A}\left(\sigma^{\mathrm{M}}, \delta\right) u^{i}+P^{S}\left(\sigma^{\mathrm{M}}, \delta\right) w^{i}+P^{q}\left(\sigma^{\mathrm{M}}, \delta\right) 0$ and, for every $s \in S$, the sum of all the components of row $s$ of $P^{A}\left(\sigma^{\mathrm{M}}, \delta\right), P^{S}\left(\sigma^{\mathrm{M}}, \delta\right)$, and $P^{q}\left(\sigma^{\mathrm{M}}, \delta\right)$ together is 1 , it follows that $\left(f^{1}\left(\sigma^{\mathrm{M}}, w\right), \ldots, f^{n}\left(\sigma^{\mathrm{M}}, w\right)\right) \in \bar{U}^{S}$.

Let $i \in N$ and $s \in S^{i}$. The correspondence $\Phi_{s}^{i}: \Sigma^{\mathrm{M}} \times \bar{U}^{S} \rightarrow \Delta\left(X^{i}\right)$ if $s=i$ and $\Phi_{s}^{i}: \Sigma^{\mathrm{M}} \times \bar{U}^{S} \rightarrow[0,1]$ if $s \in R^{i}$ is defined by taking the set of maximizers of the linear program of (4). The coefficients in the objective function are continuous in $\sigma^{\mathrm{M}}$ and $w$. By the Maximum Theorem, $\Phi_{s}^{i}$ is a non-empty, compact- and convex-valued, upper semi-continuous correspondence. Stacking all functions and correspondences together yields the correspondence $\Theta: \Sigma^{\mathrm{M}} \times \bar{U}^{S} \rightarrow \Sigma^{\mathrm{M}} \times \bar{U}^{S}$, which satisfies the conditions of Kakutani's fixed-point theorem. Hence, there exists a fixed point $\left(\sigma^{\mathrm{M} *}, w^{*}\right) \in \Sigma^{\mathrm{M}} \times \bar{U}^{S}$.

By construction of $\Theta$, each fixed point is a quasi SSPE.

\section{Proof of Proposition 8, part 1 and part 2}

For $\delta \in[0,1)$, it holds that $\left(\alpha, \beta^{-}, \beta^{+}\right)$forms an SSPE with utilities $\left(w, w^{+}, w^{-}\right)$ if and only if they are a solution to the optimality conditions (4) and the system of recursive equations (5). Part 1 and 2 of Proposition 8 can now be verified.

\section{Proof of Proposition 8, Part 3}

The system of recursive equations (5) can be written as:

$$
\begin{aligned}
w & =2 \alpha \beta^{-}+\alpha\left(1-\beta^{-}\right) w^{-}+(1-\alpha) \beta^{+}+(1-\alpha)\left(1-\beta^{+}\right) w^{+}, \\
w^{+} & =\alpha \beta^{-}+\alpha\left(1-\beta^{-}\right) w+(1-\alpha)\left(1-\beta^{+}\right) w^{-}, \\
w^{-} & =\alpha\left(1-\beta^{-}\right) w^{+}+2(1-\alpha) \beta^{+}+(1-\alpha)\left(1-\beta^{+}\right) w,
\end{aligned}
$$

and the optimality conditions (4) as: 


$$
\begin{aligned}
& 2 \beta^{-}+\left(1-\beta^{-}\right) w^{-}>\beta^{+}+\left(1-\beta^{+}\right) w^{+} \Rightarrow \alpha=1, \\
& 2 \beta^{-}+\left(1-\beta^{-}\right) w^{-}=\beta^{+}+\left(1-\beta^{+}\right) w^{+} \Rightarrow \alpha \in[0,1], \\
& 2 \beta^{-}+\left(1-\beta^{-}\right) w^{-}<\beta^{+}+\left(1-\beta^{+}\right) w^{+} \Rightarrow \alpha=0, \\
& 1>w \Rightarrow \beta^{-}=1, \\
& 1=w \Rightarrow \beta^{-} \in[0,1], \\
& 1<w \Rightarrow \beta^{-}=0, \\
& 2>w \Rightarrow \beta^{+}=1, \\
& 2=w \Rightarrow \beta^{+} \in[0,1], \\
& 2<w \Rightarrow \beta^{+}=0 .
\end{aligned}
$$

Let $\left(\alpha, \beta^{-}, \beta^{+}, w, w^{+}, w^{-}\right) \in[0,1]^{3} \times \bar{U}$ be a solution to (9)-(17). In particular, it holds that $\left(w, w^{+}, w^{-}\right) \geq 0$ and $w+w^{+}+w^{-} \leq 3$.

Claim: $\beta^{+}=1$. Suppose $\beta^{+} \in[0,1)$. It follows by (15) that $w \geq 2$, which implies that $w^{+}+w^{-} \leq 1$, so by non-negativity both $w^{+}$and $w^{-}$are less than or equal to 1 . Now (6) implies

$$
\begin{aligned}
2 & \leq w=2 \alpha \beta^{-}+\alpha\left(1-\beta^{-}\right) w^{-}+(1-\alpha) \beta^{+}+(1-\alpha)\left(1-\beta^{+}\right) w^{+} \\
& \leq \alpha\left(1+\beta^{-}\right)+(1-\alpha)=1+\alpha \beta^{-} \leq 2
\end{aligned}
$$

so both $\alpha$ and $\beta^{-}$are equal to 1 . Implication (14) yields that $w \leq 1$, a contradiction to $w \geq 2$. Consequently, the claim $\beta^{+}=1$ holds.

We substitute $\beta^{+}=1$ in (6)-(17) and establish a second claim.

Claim: $\alpha \in(0,1]$. Suppose $\alpha=0$. Now (8) implies that $w^{-}=2$, so by (9) we obtain $2=2 \beta^{-}+\left(1-\beta^{-}\right) w^{-} \leq 1$, a contradiction.

We divide the remaining cases in six classes and show that each one leads to a contradiction, with the exception of class four.

1. Suppose $\alpha \in(0,1)$ and $\beta^{-}=0$. Using (9) and (11) we obtain that $1=2 \beta^{-}+(1-$ $\left.\beta^{-}\right) w^{-}=w^{-}$. Moreover, (6)-(8) lead to

$$
\begin{aligned}
w & =1, \\
w^{+} & =\alpha w, \\
w^{-} & =\alpha w^{+}+2(1-\alpha),
\end{aligned}
$$

so $w^{+}=\alpha$ and $1=w^{-}=\alpha^{2}+2(1-\alpha)$. Solving the last equation leads to $\alpha=1$, a contradiction to $\alpha \in(0,1)$. 
2. Suppose $\alpha \in(0,1)$ and $\beta^{-} \in(0,1)$. The system of equations (6)-(8) is equal to

$$
\begin{aligned}
w & =2 \alpha \beta^{-}+\alpha\left(1-\beta^{-}\right) w^{-}+(1-\alpha), \\
w^{+} & =\alpha \beta^{-}+\alpha\left(1-\beta^{-}\right) w, \\
w^{-} & =\alpha\left(1-\beta^{-}\right) w^{+}+2(1-\alpha) .
\end{aligned}
$$

Adding up these equalities leads to

$$
w+w^{+}+w^{-}=\alpha\left(1-\beta^{-}\right)\left(w+w^{+}+w^{-}\right)+3 \alpha \beta^{-}+3-3 \alpha,
$$

so $w+w^{+}+w^{-}=3$. From $\beta^{-} \in(0,1),(12)$, and (14), we obtain that $w=1$. Substitution of $w=1$ in (7)-(8) yields

$$
\begin{aligned}
& w^{+}=\alpha \\
& w^{-}=\alpha\left(1-\beta^{-}\right) w^{+}+2(1-\alpha)=\left(1-\beta^{-}\right) \alpha^{2}+2(1-\alpha) .
\end{aligned}
$$

Adding up $w, w^{+}$, and $w^{-}$results in the equality $1+\alpha+\left(1-\beta^{-}\right) \alpha^{2}+2(1-\alpha)=3$, so $\alpha=1 /\left(1-\beta^{-}\right)>1$, a contradiction to $\alpha \in(0,1)$.

3. Suppose $\alpha \in(0,1)$ and $\beta^{-}=1$. Since $\beta^{-}=1$ we know from (14) that $w \leq 1$. At the same time we find using (6) that $w=2 \alpha+(1-\alpha)=1+\alpha>1$, leading to a contradiction.

4. Suppose $\alpha=1$ and $\beta^{-}=0$. Substitution of $\alpha=1$ and $\beta^{-}=0$ into (6)-(8) implies $w=w^{+}=w^{-}$. Next, $\alpha=1$ and $\beta^{-}=0$ combined with (11) imply $w^{-} \geq 1$, and since $w+w^{+}+w^{-} \leq 3$, we have $w=w^{+}=w^{-}=1$. It can be verified that $w=w^{+}=w^{-}=1, \alpha=1, \beta^{+}=1$ and $\beta^{-}=0$ is a solution.

5. Suppose $\alpha=1$ and $\beta^{-} \in(0,1)$. Using the same derivation as in case 2 , we find that $w+w^{+}+w^{-}=3$. Since $\beta^{-} \in(0,1)$, we find by (12) and (14) that $w=1$, so by (7) that $w^{+}=1$. Since $w+w^{+}+w^{-}=3$, we find that $w^{-}=1$. However, by (8), $w^{-}=\left(1-\beta^{-}\right) w^{+}<1$, leading to a contradiction.

6. Suppose $\alpha=1$ and $\beta^{-}=1$. By (14) it follows that $w \leq 1$, but by (6) it holds that $w=2 \beta^{-}=2$, a contradiction.

Hence, we conclude that $\alpha=1, \beta^{+}=1$, and $\beta^{-}=0$ together with $w=w^{+}=$ $w^{-}=1$ is the unique quasi SSPE.

The unique quasi SSPE is the only candidate for an SSPE. Since $\alpha=1$ and $\beta^{-}=0$ leads to perpetual disagreement, we have $v=v^{+}=v^{-}=0$. Since a proposer has a profitable one-stage deviation to $\alpha=0$, the quasi SSPE is not an SSPE.

\section{Proof of Proposition 9.}

Let $i \in N$. By (3), we have that

$$
P^{S}\left(\sigma^{\mathrm{M}}, \delta\right) v^{i}\left(\sigma^{\mathrm{M}}, \delta\right)=\left[\sum_{\tau=2}^{\infty} P^{S}\left(\sigma^{\mathrm{M}}, \delta\right)^{\tau-1}\right] P^{A}\left(\sigma^{\mathrm{M}}, \delta\right) u^{i}=v^{i}\left(\sigma^{\mathrm{M}}, \delta\right)-P^{A}\left(\sigma^{\mathrm{M}}, \delta\right) u^{i}
$$


By rewriting this equation, we obtain the stated result.

\section{Proof of Proposition 10.}

The matrix $P^{S}\left(\sigma^{\mathrm{M}}, \delta\right)$ is non-negative with row sums less than or equal to 1 . Let $\lambda\left(P^{S}\left(\sigma^{\mathrm{M}}, \delta\right)\right)$ denote an eigenvalue of $P^{S}\left(\sigma^{\mathrm{M}}, \delta\right)$ with the largest absolute value. It follows from Solow (1952) that

1. $\lambda\left(P^{S}\left(\sigma^{\mathrm{M}}, \delta\right)\right) \in[0,1]$ is real.

2. The sum $\sum_{\tau=0}^{\infty} P^{S}\left(\sigma^{\mathrm{M}}, \delta\right)^{\tau}$ exists if and only if $\lambda\left(P^{S}\left(\sigma^{\mathrm{M}}, \delta\right)\right)<1$.

3. If $\lambda\left(P^{S}\left(\sigma^{\mathrm{M}}, \delta\right)\right)<1$, then the inverse $\left[I-P^{S}\left(\sigma^{\mathrm{M}}, \delta\right)\right]^{-1}=\sum_{\tau=0}^{\infty} P^{S}\left(\sigma^{\mathrm{M}}, \delta\right)^{\tau}$ exists and is non-negative.

For our bargaining model, 3. implies

$$
v^{i}\left(\sigma^{\mathrm{M}}, \delta\right)=\sum_{\tau=1}^{\infty} P^{S}\left(\sigma^{\mathrm{M}}, \delta\right)^{\tau-1} P^{A}\left(\sigma^{\mathrm{M}}, \delta\right) u^{i}=\left[I-P^{S}\left(\sigma^{\mathrm{M}}, \delta\right)\right]^{-1} P^{A}\left(\sigma^{\mathrm{M}}, \delta\right) u^{i}
$$

The following result is now immediate:

A. If $\lambda\left(P^{S}\left(\sigma^{\mathrm{M}}, \delta\right)\right)<1$, then $v^{i}\left(\sigma^{\mathrm{M}}, \delta\right)$ is the unique solution to the system of recursive equations $(5)$.

The results in Solow (1952) relate conditions on $\lambda\left(P^{S}\left(\sigma^{\mathrm{M}}, \delta\right)\right)$ to row sums associated with the irreducible blocks $\tilde{P}_{e e}\left(\sigma^{\mathrm{M}}, \delta\right)$ for $e=1, \ldots, f$ of a finest decomposition of $P^{S}\left(\sigma^{\mathrm{M}}, \delta\right)$. We obtain the following results:

B. $\lambda\left(\tilde{P}_{e e}\left(\sigma^{\mathrm{M}}, \delta\right)\right)=1$ if and only if all row sums of $\tilde{P}_{e e}\left(\sigma^{\mathrm{M}}, \delta\right)$ are equal to 1 .

C. $\lambda\left(\tilde{P}_{e e}\left(\sigma^{\mathrm{M}}, \delta\right)\right)<1$ if and only if at least one row sum of $\tilde{P}_{e e}\left(\sigma^{\mathrm{M}}, \delta\right)$ is less than 1 .

The set of eigenvalues of $P^{S}\left(\sigma^{\mathrm{M}}, \delta\right)$ is equal to the union of the sets of eigenvalues of $\tilde{P}_{e e}\left(\sigma^{\mathrm{M}}, \delta\right)$ for $e=1, \ldots, f$. The result now follows.

Proof of Proposition 11. Let $\tilde{P}\left(\sigma^{\mathrm{M}}, \delta\right)$ be a finest decomposition of $P^{S}\left(\sigma^{\mathrm{M}}, \delta\right)$. By Proposition 10, we have to show that, for every $e \in\{1, \ldots, f\}$, the irreducible block $\tilde{P}_{e e}\left(\sigma^{\mathrm{M}}, \delta\right)$ has at least one row sum less than 1 .

Suppose there is $e \in\{1, \ldots, f\}$ such that every row sum of $P_{e e}\left(\sigma^{\mathrm{M}}, \delta\right)$ is equal to 1 . The Markov process never leaves $S_{e}\left(\sigma^{\mathrm{M}}, \delta\right)$, so this set has to contain at least one proposer state, say $i \in S$. For every $x=(a, o) \in X^{i}$ such that $\alpha^{i}(x)>0$, it also holds that $(i, x, 2) \in S_{e}\left(\sigma^{\mathrm{M}}, \delta\right)$. Moreover, for every $j \in C(o)$ such that $k^{j}(o) \leq$ $|C(o)|,\left(i, x, k^{j}(o)\right) \in S_{e}\left(\sigma^{\mathrm{M}}, \delta\right)$ and $\beta^{j}\left(i, x, k^{j}(o)\right)>0$ implies $\left(i, x, k^{j}(o)+1\right) \in$ $S_{e}\left(\sigma^{\mathrm{M}}, \delta\right)$. In case there is $x=(a, o) \in X^{i}$ such that $\alpha^{i}(x)>0$ and, for every $j \in C(o), \beta^{j}\left(i, x, k^{j}(o)\right)>0$, then with positive probability there is a transition to the absorbing state $a$, a state outside $S_{e}\left(\sigma^{\mathrm{M}}, \delta\right)$, leading to a contradiction. It follows that, for every $x=(a, o) \in X^{i}$ such that $\alpha^{i}(x)>0$, there is $j \in C(o)$ such that $\beta^{j}\left(i, x, k^{j}(o)\right)=0$. Using the assumptions of the proposition, it follows that $\delta<1$. Since any proposal that $i$ makes with positive probability is rejected, there is a transition to the absorbing state $q$ with positive probability $1-\delta$, a state outside $S_{e}\left(\sigma^{\mathrm{M}}, \delta\right)$, leading to a contradiction.

Proof of Proposition 12. Let $\tilde{P}\left(\sigma^{\mathrm{M}}, \delta\right)$ be a finest decomposition of $P^{S}\left(\sigma^{\mathrm{M}}, \delta\right)$.

\section{$\Rightarrow$}


By Proposition 10, for every $e \in\{1, \ldots, f\}$, the irreducible block $\tilde{P}_{e e}\left(\sigma^{\mathrm{M}}, \delta\right)$ has at least one row sum less than 1 .

Suppose $\delta=1$ and, for every $i \in N$, for every $x=(a, o) \in X^{i}$ such that $\alpha^{i}(x)>0$, there is $j \in C(o)$ such that $\beta^{j}\left(i, x, k^{j}(o)\right)=0$. Let $i^{1} \in S$ be an arbitrary initial state. Our supposition implies that there is perpetual disagreement when starting from $i^{1}$. Let $e \in\{1, \ldots, f\}$ be such that $i^{1} \in S_{e}\left(\sigma^{\mathrm{M}}, \delta\right)$. Then every row sum of $\tilde{P}_{e e}\left(\sigma^{\mathrm{M}}, \delta\right)$ is equal to 1 , leading to a contradiction. Consequently, it holds that $\delta<1$ or there is $i^{\prime} \in N$ and $x^{\prime}=\left(a^{\prime}, o^{\prime}\right) \in X^{i^{\prime}}$ such that $\alpha^{i^{\prime}}\left(x^{\prime}\right)>0$ and, for every $j \in C\left(o^{\prime}\right)$, $\beta^{j}\left(i^{\prime}, x^{\prime}, k^{j}\left(o^{\prime}\right)\right)>0$.

$\Leftarrow$

By Proposition 10, we have to show that, for every $e \in\{1, \ldots, f\}$, the irreducible block $\tilde{P}_{e e}\left(\sigma^{\mathrm{M}}, \delta\right)$ has at least one row sum less than 1 .

Suppose there is $e \in\{1, \ldots, f\}$ such that every row sum of $P_{e e}\left(\sigma^{\mathrm{M}}, \delta\right)$ is equal to 1 . The Markov process never leaves $S_{e}\left(\sigma^{\mathrm{M}}, \delta\right)$, so this set has to contain at least one proposer state, say $i \in S$. For every $x=(a, o) \in X^{i}$ such that $\alpha^{i}(x)>0$, it also holds that $(i, x, 2) \in S_{e}\left(\sigma^{\mathrm{M}}, \delta\right)$. Moreover, for every $j \in C(o)$ such that $k^{j}(o) \leq$ $|C(o)|,\left(i, x, k^{j}(o)\right) \in S_{e}\left(\sigma^{\mathrm{M}}, \delta\right)$ and $\beta^{j}\left(i, x, k^{j}(o)\right)>0$ implies $\left(i, x, k^{j}(o)+1\right) \in$ $S_{e}\left(\sigma^{\mathrm{M}}, \delta\right)$. Any $x=(a, o) \in X^{i}$ such that $\alpha^{i}(x)>0$ is rejected, since otherwise there would be a transition to the absorbing state $a$, a state outside $S_{e}\left(\sigma^{\mathrm{M}}, \delta\right)$, leading to a contradiction. We obtain $\delta=1$, since otherwise there would be a transition to the absorbing state $q$ with positive probability $1-\delta$, a state outside $S_{e}\left(\sigma^{\mathrm{M}}, \delta\right)$.

For every $i \in N$, choose $x^{i}=\left(a^{i}, o^{i}\right) \in X^{i}$ such that $\alpha^{i}\left(x^{i}\right)>0$ and let $r^{i} \in C(o)$ be the lowest ranked player in $o$ such that $\beta^{r^{i}}\left(a^{i}, o^{i}, k^{r^{i}}\right)=0$. Since the bargaining protocol is irreducible, it follows that the matrix

$$
\left[\begin{array}{ccc}
\rho_{1}\left(1, x^{1}, r^{1}\right) & \cdots & \rho_{1}\left(n, x^{n}, r^{n}\right) \\
\vdots & \vdots \\
\rho_{n}\left(1, x^{1}, r^{1}\right) & \cdots & \rho_{n}\left(n, x^{n}, r^{n}\right)
\end{array}\right]
$$

is irreducible, so in particular $i^{\prime} \in S_{e}\left(\sigma^{\mathrm{M}}, \delta\right),\left(i^{\prime},\left(a^{\prime}, o^{\prime}\right), 2\right) \in S_{e}\left(\sigma^{\mathrm{M}}, \delta\right)$ and, for every $j \in C\left(o^{\prime}\right),\left(i^{\prime}, x^{\prime}, k^{j}\left(o^{\prime}\right)\right) \in S_{e}\left(\sigma^{\mathrm{M}}, \delta\right)$. Since the proposal $x^{\prime}$ is accepted with positive probability, the absorbing state $a^{\prime}$ is reached with positive probability, a state outside $S_{e}\left(\sigma^{\mathrm{M}}, \delta\right)$, leading to a contradiction. Consequently, for every $e \in\{1, \ldots, f\}$, at least one row sum of $P_{e e}\left(\sigma^{\mathrm{M}}, \delta\right)$ is less than one.

\section{Proof of Proposition 13.}

\section{$\Rightarrow$}

Since states in $S^{\mathrm{pd}}\left(\sigma^{\mathrm{M}}, \delta\right)$ lead to perpetual disagreement, we have, for every $s \in$ $S^{\mathrm{pd}}\left(\sigma^{\mathrm{M}}, \delta\right), v^{i}\left(s ; \sigma^{\mathrm{M}}, \delta\right)=0$. From $w^{i}=v^{i}\left(\sigma^{\mathrm{M}}, \delta\right)$, it follows that, for every $s \in$ $S^{\mathrm{pd}}\left(\sigma^{\mathrm{M}}, \delta\right), w^{i}(s)=0$.

$\Leftarrow$

We define $S^{\prime}=S \backslash S^{\mathrm{pd}}\left(\sigma^{\mathrm{M}}, \delta\right)$. We now define the Markov process on $S^{\prime} \cup A \cup$ $\{q\}$ induced by $\sigma^{\mathrm{M}}$ that is obtained by identifying all states in $S^{\mathrm{pd}}\left(\sigma^{\mathrm{M}}, \delta\right)$ with the absorbing state $q$. 
Let $s \in S^{\prime}$. For every $\tilde{s} \in S^{\prime}$, we define $Q_{s \widetilde{s}}^{S^{\prime}}\left(\sigma^{\mathrm{M}}, \delta\right)=P_{s \tilde{s}}^{S}\left(\sigma^{\mathrm{M}}, \delta\right)$, for every $a \in A$, we define $Q_{s a}^{A}\left(\sigma^{\mathrm{M}}, \delta\right)=P_{s a}^{A}\left(\sigma^{\mathrm{M}}, \delta\right)$, and $Q_{s q}^{q}\left(\sigma^{\mathrm{M}}, \delta\right)=P_{s q}^{q}\left(\sigma^{\mathrm{M}}, \delta\right)+$ $\sum_{s^{\prime} \in S^{\mathrm{pd}}\left(\sigma^{\mathrm{M}}, \delta\right)} P_{s s^{\prime}}^{q}\left(\sigma^{\mathrm{M}}, \delta\right)$. The transition probabilities of the Markov process on $S^{\prime} \cup$ $A \cup\{q\}$ are then given by

$$
\Lambda^{\prime}\left(\sigma^{\mathrm{M}}, \delta\right)=\left[\begin{array}{ccc}
Q^{S^{\prime}}\left(\sigma^{\mathrm{M}}, \delta\right) & Q^{A}\left(\sigma^{\mathrm{M}}, \delta\right) & Q^{q}\left(\sigma^{\mathrm{M}}, \delta\right) \\
0 & I & 0 \\
0 & 0 & 1
\end{array}\right]
$$

Let $w^{i}$ be a solution to the system of recursive equations (5) with $w^{i}(s)=0$ if $s \in S^{\mathrm{pd}}\left(\sigma^{\mathrm{M}}, \delta\right)$. Define $\tilde{w}^{i}=\left(w^{i}(s)\right)_{s \in S^{\prime}}$ and $\tilde{v}^{i}\left(\sigma^{\mathrm{M}}, \delta\right)=\left(v^{i}\left(s ; \sigma^{\mathrm{M}}, \delta\right)\right)_{s \in S^{\prime}}$.

For every $s \in S^{\prime}$, we have that

$$
\begin{aligned}
Q_{s \cdot}^{A}\left(\sigma^{\mathrm{M}}, \delta\right) u^{i}+Q_{s \cdot}^{S^{\prime}}\left(\sigma^{\mathrm{M}}, \delta\right) \tilde{w}^{i} & =P_{s \cdot}^{A}\left(\sigma^{\mathrm{M}}, \delta\right) u^{i}+\sum_{\tilde{s} \in S^{\prime}} P_{s \tilde{s}}^{S}\left(\sigma^{\mathrm{M}}, \delta\right) \tilde{w}^{i} \\
& =P_{s \cdot}^{A}\left(\sigma^{\mathrm{M}}, \delta\right) u^{i}+P_{s \cdot}^{S}\left(\sigma^{\mathrm{M}}, \delta\right) w^{i} \\
& =w^{i}(s)
\end{aligned}
$$

where the first equality follows by definition, the second equality since, for every $s \in S^{\mathrm{pd}}\left(\sigma^{\mathrm{M}}, \delta\right), w^{i}(s)=0$, and the third equality since $w^{i}$ is a solution to the system of recursive equations (5). We have shown that

$$
\tilde{w}^{i}=Q^{A}\left(\sigma^{\mathrm{M}}, \delta\right) u^{i}+Q^{S^{\prime}}\left(\sigma^{\mathrm{M}}, \delta\right) \tilde{w}^{i}
$$

Since by construction, all irreducible blocks in a finest decomposition $\tilde{Q}\left(\sigma^{\mathrm{M}}, \delta\right)$ of $Q^{S}\left(\sigma^{\mathrm{M}}, \delta\right)$ have at least one row sum less than $1, \tilde{w}^{i}$ is the only solution to (18).

It holds that

$$
\begin{aligned}
Q_{s .}^{A}\left(\sigma^{\mathrm{M}}, \delta\right) u^{i}+Q_{s \cdot}^{S^{\prime}}\left(\sigma^{\mathrm{M}}, \delta\right) \tilde{v}^{i}\left(\sigma^{\mathrm{M}}, \delta\right) & =P_{s \cdot}^{A}\left(\sigma^{\mathrm{M}}, \delta\right) u^{i}+\sum_{\tilde{s} \in S^{\prime}} P_{s \tilde{s}^{S}}^{S}\left(\sigma^{\mathrm{M}}, \delta\right) \tilde{v}^{i}\left(\sigma^{\mathrm{M}}, \delta\right) \\
& =P_{s \cdot}^{A}\left(\sigma^{\mathrm{M}}, \delta\right) u^{i}+P_{s \cdot}^{S}\left(\sigma^{\mathrm{M}}, \delta\right) v^{i}\left(\sigma^{\mathrm{M}}, \delta\right) \\
& =\tilde{v}^{i}\left(s ; \sigma^{\mathrm{M}}, \delta\right),
\end{aligned}
$$

where the first equality follows by definition, the second equality since, for every $s \in$ $S^{\mathrm{pd}}\left(\sigma^{\mathrm{M}}, \delta\right), \tilde{v}^{i}\left(s ; \sigma^{\mathrm{M}}, \delta\right)=0$, and the third equality since $\tilde{v}^{i}\left(\sigma^{\mathrm{M}}, \delta\right)$ is a solution to the system of recursive equations (5). It follows that $\tilde{w}^{i}=\tilde{v}^{i}\left(\sigma^{\mathrm{M}}, \delta\right)$. For $s \in S^{\mathrm{pd}}\left(\sigma^{\mathrm{M}}, \delta\right)$, we have that $w^{i}(s)=v^{i}\left(s ; \sigma^{\mathrm{M}}, \delta\right)=0$. We have shown that $w^{i}=v^{i}\left(\sigma^{\mathrm{M}}, \delta\right)$.

Proof of Proposition 14. Existence follows from standard arguments for $\delta \in[0,1)$. For $\delta=1$, existence follows from Theorem 7.1.9 in Puterman (1994) since the state and action space is finite for every player and utilities are non-negative and bounded. 
If $\delta \in[0,1)$, then $S^{\mathrm{pd}}\left(\sigma^{\mathrm{M}}, \delta\right)=\varnothing$, and we obtain the standard characterization. For $\delta=1$, iterated substitution of $w^{i}$ in (5) yields

$$
w^{i}=\sum_{\tau=1}^{T} P^{S}\left(\sigma^{\mathrm{M}}, \delta\right)^{\tau-1} P^{A}\left(\sigma^{\mathrm{M}}, \delta\right) u^{i}+P^{S}\left(\sigma^{\mathrm{M}}, \delta\right)^{T} w^{i}
$$

Then, $w^{i}=v^{i}\left(\sigma^{\mathrm{M}}, \delta\right)$ if and only if

$$
\lim _{T \rightarrow \infty} P^{S}\left(\sigma^{\mathrm{M}}, \delta\right)^{T} w^{i}=0
$$

By Proposition 13, it holds that $w^{i}=v^{i}\left(\sigma^{\mathrm{M}}, \delta\right)$ if and only if, for every $s \in$ $S^{\mathrm{pd}}\left(\sigma^{\mathrm{M}}, \delta\right), w^{i}(s)=0$. We conclude that $\lim _{T \rightarrow \infty} P^{S}\left(\sigma^{\mathrm{M}}, \delta\right)^{T} w^{i}=0$. The characterization now follows from Theorem 7.1.7 in Puterman (1994).

\section{References}

Alós-Ferrer, C., Ritzberger, K.: Equilibrium existence for large perfect information games. J. Math. Econ. 62, 5-18 (2016)

Alós-Ferrer, C., Ritzberger, K.: Does backwards induction imply subgame perfection? Games Econ. Behav. 103, 19-29 (2017)

Banks, J., Duggan, J.: A bargaining model of collective choice. Am. Polit. Sci. Rev. 94, 73-88 (2000)

Banks, J., Duggan, J.: A general bargaining model of legislative policy-making. Q. J. Polit. Sci. 1, 49-85 (2006)

Baron, D.P., Ferejohn, J.A.: Bargaining in legislatures. Am. Polit. Sci. Rev. 83, 1181-1206 (1989)

Binmore, K.: Bargaining and coalitions. In: Roth, A. (ed.) Game Theoretic Models of Bargaining, pp. 269-304. Cambridge University Press, Cambridge (1986)

Binmore, K.: Perfect equilibria in bargaining models. In: Binmore, K., Dasgupta, P. (eds.) The Economics of Bargaining, pp. 77-105. Basil Blackwell, Oxford (1987)

Binmore, K., Rubinstein, A., Wolinsky, A.: The Nash bargaining solution in economic modelling. RAND J. Econ. 17, 176-188 (1986)

Blackwell, D., Ferguson, T.: The big match. Ann. Math. Stat. 39, 159-163 (1968)

Bloch, F.: Sequential formation of coalitions in games with externalities and fixed payoff division. Games Econ. Behav. 14, 90-123 (1996)

Bloch, F., Diamantoudi, E.: Noncooperative formation of coalitions in hedonic games. Int. J. Game Theory 40, 263-280 (2011)

Britz, V., Herings, P.J.J., Predtetchinski, A.: Non-cooperative support for the asymmetric Nash bargaining solution. J. Econ. Theory 145, 1951-1967 (2010)

Chatterjee, K., Dutta, B., Ray, D., Sengupta, K.: A noncooperative theory of coalitional bargaining. Rev. Econ. Stud. 60, 463-477 (1993)

Dàvila, J., Eeckhout, J.: Competitive bargaining equilibrium. J. Econ. Theory 139, 269-294 (2008)

Duggan, J.: Existence of stationary bargaining equilibria. Games Econ. Behav. 102, 111-126 (2017)

Eraslan, H., Evdokimov, K.: Legislative and multilateral bargaining. Annu. Rev. Econ. 11, 443-472 (2019)

Fink, A.M.: Equilibrium in a stochastic $n$-person game. J. Sci. Hiroshima Univ. Ser. A I 28, 89-93 (1964)

Flesch, J., Kuipers, J., Schoenmakers, G., Vrieze, K.: Subgame perfection in positive recursive games with perfect information. Math. Oper. Res. 35, 193-207 (2010)

Haller, H.: Non-cooperative bargaining of $N \geq 3$ players. Econ. Lett. 22, 11-13 (1986)

Harris, C.: Existence and characterization of perfect equilibrium in games of perfect information. Econometrica 53, 613-628 (1985)

Herings, P.J.J., Houba, H.: The condorcet paradox revisited. Soc. Choice Welf. 47, 141-186 (2016)

Herings, P.J.J., Meshalkin, A., Predtetchinski, A.: A one-period memory folk theorem for multilateral bargaining games. Games Econ. Behav. 103, 185-198 (2017) 
Herrero, M.J.: A Strategic Bargaining approach to market institutions. PhD Thesis, London School of Economics, pp. 1-111 (1985)

Horniaček, M.: Negotiation, preferences over agreements, and the core. Int. J. Game Theory 37, 235-249 (2008)

Houba, H., Bolt, W.: Credible Threats in Negotiations, A Game-theoretic Approach, Theory and Decision Library, Series C: Game Theory, Mathematical Programming and Operations Research, vol. 32. Kluwer, Norwell (2002)

Kalandrakis, T.: Equilibria in sequential bargaining games as solutions to systems of equations. Econ. Lett. 84, 407-411 (2004a)

Kalandrakis, T.: A three-player dynamic majoritarian bargaining game. J. Econ. Theory 116, 294-322 (2004b)

Kuipers, J., Flesch, J., Schoenmakers, G., Vrieze, K.: Subgame-perfection in recursive perfect information games. Econ. Theor. 71, 603-662 (2021)

Livshits, I.: On non-existence of pure strategy Markov perfect equilibrium. Econ. Lett. 76, 393-396 (2002)

Magill, M., Quinzii, M.: Indeterminacy of equilibrium in stochastic OLG models. Econ. Theor. 21, 435-454 (2003)

Maskin, E.S., Tirole, J.: Markov perfect equilibrium, I. Observable actions. J. Econ. Theory 100, 191-219 (2001)

Mertens, J.-F., Neyman, A.: Stochastic games. Int. J. Game Theory 10, 53-66 (1981)

Moldovanu, B., Winter, E.: Order independent equilibria. Games Econ. Behav. 9, 21-34 (1995)

Muthoo, A.: A note on bargaining over a finite number of feasible agreements. Econ. Theor. 1, 290-292 (1991)

Muthoo, A.: Bargaining Theory with Applications. Cambridge University Press, Cambridge (1999)

Osborne, M.J., Rubinstein, A.: Bargaining and Markets. Academic Press, San Diego (1990)

Perry, M., Reny, P.J.: A noncooperative view of coalition formation and the core. Econometrica 62, 795-817 (1994)

Puterman, M.L.: Markov Decision Processes, Discrete Stochastic Dynamic Programming. Wiley, Hoboken (1994)

Ray, D.: A Game-Theoretic Perspective on Coalition Formation. Oxford University Press, Oxford (2007)

Rubinstein, A.: Perfect equilibrium in a bargaining model. Econometrica 50, 97-109 (1982)

Selten, R.: A noncooperative model of characteristic function bargaining. In: Böhm, V., Nachtkamp, H.H. (eds.), Essays in Game Theory and Mathematical Economics in Honor of Oskar Morgenstern. Bibliografisches Institut Mannheim, pp. 131-151 (1981)

Sobel, M.J.: Noncooperative stochastic games. Ann. Math. Stat. 42, 1930-1935 (1971)

Solow, R.M.: On the structure of linear models. Econometrica 20, 29-46 (1952)

Takahashi, M.: Equilibrium points of stochastic non-cooperative $n$-person games. J. Sci. Hiroshima Univ. Ser. A I 28, 95-99 (1964)

Vieille, N.: Two-player stochastic games I: a reduction. Israel J. Math. 119, 55-91 (2000a)

Vieille, N.: Two-player stochastic games II: the case of recursive games. Israel J. Math. 119, 93-126 (2000b)

Vieille, N.: Small perturbations and stochastic games. Israel J. Math. 119, 127-142 (2000c)

Publisher's Note Springer Nature remains neutral with regard to jurisdictional claims in published maps and institutional affiliations. 